\title{
TOWARDS AN IMPROVED HELIUM-BASED REFRATOMETER FOR PRESSURE MEASUREMENTS
}

\author{
Z. Silvestri ${ }^{1}$, D. Bentouati ${ }^{2}$, P. Otal ${ }^{3}$ and J-P. Wallerand ${ }^{4}$ \\ ${ }^{1}$ LCM LNE/Cnam, 1 rue Gaston Boissier, Paris, France zaccaria.silvestri@cnam.fr \\ ${ }^{2}$ LCM LNE/Cnam, 1 rue Gaston Boissier, Paris, France djilali.bentouati@lne.fr \\ ${ }^{3}$ LCM LNE/Cnam, 1 rue Gaston Boissier, Paris, France pierre.otal@lne.fr \\ ${ }^{4}$ LCM LNE/Cnam, 1 rue Gaston Boissier, Paris, France jean-pierre.wallerand@cnam.fr
}

\begin{abstract}
:
This paper describes a quantum realization of the pascal based a helium absolute refractometer at $532 \mathrm{~nm}$. The short-term stability in pressure is \pm 2 $\mathrm{mPa}$ with a resolution in pressure of this new optical pressure standard is better than $1 \mathrm{mPa}$ with temperature stability about $1 \mathrm{mK}$. A new design of the refractometer is presented to have better temperature stability and accurate temperature measurements to reach uncertainties in pressure better than current conventional methods.
\end{abstract}

Keywords: refractometry, Fabry-Perot, pressure measurement, quantum pascal

\section{INTRODUCTION}

For many decades and without any significant expected improvement, primary absolute pressure measurements in the range $1 \mathrm{~Pa}$ to $100 \mathrm{kPa}$ are mainly based on liquid column manometers, gas-operated pressure balance or static expansions systems. The relative uncertainty in CMCs is about $10^{-6}$ at $100 \mathrm{kPa}$ and about $10^{-4}$ at $1 \mathrm{~Pa}$.

At LCM, over the past few decades, the best realizations of the pascal, see Figure 1, have been obtained using two different standards. First one in the range from $10 \mathrm{kPa}$ to $500 \mathrm{kPa}$, an automatic absolute pressure balance APX50 equipped with $50 \mathrm{~mm}$ diameter piston-cylinder units is used. For the realization and the dissemination of the pressure scale, in the range $0.5 \mathrm{~Pa}$ to $10 \mathrm{kPa}$ is achieved with a force-balanced piston gauge (FPG). For example, the extended relative uncertainty is $9 \times 10^{-6}$ at $100 \mathrm{kPa}$ and $6 \times 10^{-4}$ at $1 \mathrm{kPa}$. Below $500 \mathrm{~Pa}$, the relative uncertainty increases sharply.

There is a special need to reduce relative uncertainties below $100 \mathrm{kPa}$ with, if possible, a single instrument in a wide range of pressures below atmospheric pressure. The Consultative Committee for Masses and Associated Quantities (CCM) and the Euramet Technical Committee for Masses and
Associated Quantities (TC-M) have also identified the needs and limitations of conventional methods.

With the new international system of units (SI), the scientific community is exploring new avenues directly linked to fundamental laws and or constants of physics. Thus, for pascal, a standard could be based on optical or electrical measurements such as the refractive index or the permittivity of a gas, certain parameters of which can be calculated ab initio (with helium for example). In this context, it is necessary to continue the scientific and technological development of these methods to extend the measurement range and significantly the uncertainties of conventional methods.

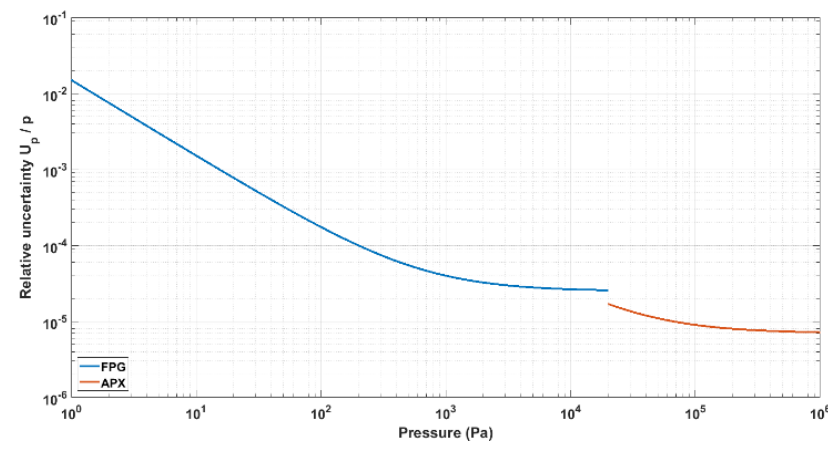

Figure 1: Current pressure relative uncertainties at LCM. In the range $10 \mathrm{kPa}$ to $500 \mathrm{kPa}$, an automatic absolute pressure balance APX50 and in the range $0.5 \mathrm{~Pa}$ to $10 \mathrm{kPa}$ a force-balanced piston gauge (FPG).

With the new international system of units (SI), many developments are now in progress for the realization of a "quantum pascal" by means of methods such as superconductive microwave resonators [1], Rayleigh scattering, multi-reflection interferometry [2], gas phase thermometry and absorption spectroscopy methods [3] as well as Fabry-Pérot (FP) cavity refractometers [4].

In the field of optical methods based on FabryPérot, the National Institute of Standards and Technology (NIST) develop since 2004 an absolute refractometer based on a double Fabry-Pérot cavity in ULE using He-Ne lasers. NIST claims an extended 
relative uncertainty $\mathrm{U}_{r}(p)=18 \times 10^{-6}$ at $100 \mathrm{kPa}$ and $4 \times 10^{-3}$ at $1 \mathrm{~Pa}$ [5], [6].

RISE (RISE Research Institutes of Sweden) and Umeå university in Sweden also develop from 2000's, this type of refractometer (double FP cavity in Zerodur $^{\circledR}$ ) using an original method of pressure modulation (GAMOR) to limit temperature effects on the cavity [7], [8]. NIM (National Institute of Metrology of China) in China, NMIJ (National Metrology Institute of Japan) in Japan [9] and PTB (Physikalisch-Technische Bundesanstalt) in Germany [10] start the development of such instruments.

French metrology is currently developing a quantum realization of the pascal based on a helium absolute refractometer at $532 \mathrm{~nm}$ [11]: the optical path travelled by a laser beam in a single Fabry-Perot cavity is measured either with helium gas or under vacuum. The ratio between these two optical paths, with appropriate corrections, gives the refractivity of the helium. By comparison with an ab-initio determination of helium refractive index and an accurate measurement of the gas temperature, we determine the absolute gas pressure inside the refractometer using Lorentz-Lorenz equation. Other gases can be used, after determining their atomic parameters by comparison with helium.

This paper describes shortly the current experimental set-up and the latest results on the performance of the refractometer for pressure measurements in the range $100 \mathrm{~Pa}$ to $100 \mathrm{kPa}$. We also present an improved version to insure the best stability in temperature, a limited temperature gradient and accurate temperature measurements with an uncertainty lower than $1 \mathrm{mK}$.

\section{EXPERIMENTAL SET-UP}

\subsection{General view}

The experimental set-up was formerly used as an absolute refractometer of air and helium [12] for the determination of refractive index with a standard uncertainty of $10^{-8}$. It is mainly composed of a large vacuum chamber that hosts the Fabry-Perot cavity and two frequency doubled Nd:YAG lasers at $532 \mathrm{~nm}$ : one is servo-controlled on transition iodine and second one is servo-controlled on the FP cavity. A frequency beat is performed between the two lasers to measure the frequency of second laser.

The Fabry-Perot cavity in Figure 2 is a planoconcave optical resonator which spacer is made of Zerodur $^{\circledR}$ of $100 \mathrm{~mm}$-long and $25 \mathrm{~mm}$-diameter. It is placed inside a copper and dural cylinders with a temperature regulated system using thermofoils. The assembly is then placed in a $10 \mathrm{~L}$ vacuum enclosure. Gas temperature is measured with 4 wires $100 \Omega$ RTD sensors along the FPM cavity.

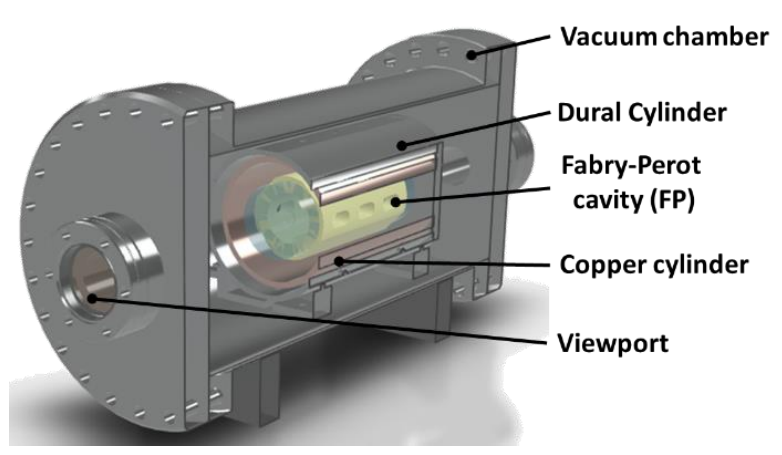

Figure 2: Cross-section of the vacuum chamber with a Fabry-Perot cavity. Fabry-Perot resonator composed of two silica mirrors and a spacer made in Zerodur ${ }^{\circledR}$.

\subsection{Methods to pressure determination}

Pressure $P$ can be determined using two methods (Figure 3). In a first approach, refractometer can be used in absolute way: starting from vacuum as reference and measuring frequency variation $v$ of the servo-controlled laser on the cavity between vacuum and pressure to measure. Frequency variation allows to determine refractive index $n$ variation of the gas and therefore pressure variation if the thermodynamic temperature $T$ is accurately measured.

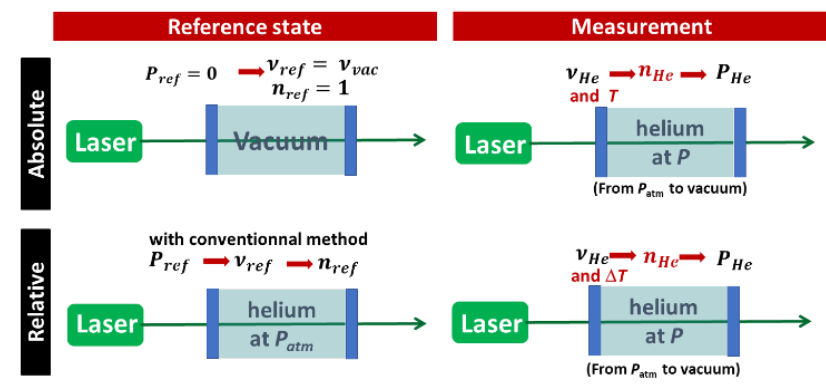

Figure 3: Schematic of the two methods (absolute and relative) to determine a pressure using a refractometer. $P$ is a pressure, $n$ is the refractive index et $v$ the frequency. $r e f$ index indicates the reference state of the parameters and $H e$ index indicates the measurement state.

Second way consist to use the refractometer in relative mode. In this case, reference state is a gas near the atmospheric pressure. This pressure is measured with a conventional method (uncertainty better than $10^{-5}$ ). Then, frequency variation of the laser is measured between this reference pressure and pressures up to $100 \mathrm{~Pa}$. It is not necessary to measure thermodynamic temperature of the gas but just only the temperature difference. In this case, the traceability of the pressure is given by the pressure balance for the absolute value and by atomic gas parameters for pressure variation.

\section{MAIN RESULTS}

To achieve the final objectives in terms of pressure uncertainties $\left(5 \times 10^{-4}\right.$ at $1 \mathrm{~Pa}, 10^{-5}$ à $\left.1 \mathrm{kPa}\right)$, ageing and mechanical deformation of the FP cavity 
are two parameters which limit the uncertainty in relative mode [11]. In the case of absolute use, the thermodynamic temperature of the gas is also a limiting factor. We need to measure the temperature helium gas with an uncertainty lower than $1 \mathrm{mK}$ and to maintain inside the refractometer a pressure with a stability lower than $0,01 \mathrm{~Pa}$ over several tens of seconds.

\subsection{Ageing, $x x x$ and uncertainties}

The uncertainty of the ageing of the cavity is $1 \times 10^{-11} /$ day . This value leads to a pressure variation of $3 \mathrm{mPa}$ for $\mathrm{N}_{2}$ and $20 \mathrm{mPa}$ for $\mathrm{He}$. It is necessary to improve the uncertainty on this parameter by a factor greater than three for pressure below $100 \mathrm{~Pa}$ by using a pressure balance.

For the thermal expansion coefficient, a relative uncertainty about $4 \%$ is enough if the temperature of the cavity can be reproduced at $10 \mathrm{mK}$. These two conditions are already met today with a relative uncertainty of $0,15 \mathrm{mPa}$ for $\mathrm{N}_{2}$ and $1,2 \mathrm{mPa}$ for He.

The uncertainty of $5 \times 10^{-15}$ obtained for the pressure-induced deformation for the absolute mode (vacuum as a reference) do not ensure the required uncertainty. In the case of the relative mode measurements, the uncertainty is sufficient. However, recent changes to the cavity mirrors have significantly improved the uncertainty on the measurement of the free spatial range (about $200 \mathrm{~Hz}$ in vacuum) and should improve this uncertainty component.

\subsection{Temperature stability and measurement of the temperature}

To stabilize the temperature in the FP cavity, we therefore set up a temperature regulation system at $\pm 1 \mathrm{mK}$ (completely autonomous system based on Arduino). For that, to the Dural cylinder, we are glued two thermofoils in the opposite side. The copper cylinder allows to create a heat shield. Finally, the entire enclosure was confined in an expanded polystyrene box. The temperature is measured inside the cavity by 4 Pt100 RTD probes (4-wire measurement) distributed over the entire cavity.

The temperature stability inside the cavity is about $2 \mathrm{mK}$ but the temperature gradient along the $100 \mathrm{~mm}$ of the cavity is around $5 \mathrm{mK}$. Long-term stability is also not guaranteed due to the excessive volume of the vacuum enclosure.

With the Pt100 probes, it is not possible to measure thermodynamic temperature better than $10 \mathrm{mK}$. For an absolute pressure measurement with a relative uncertainty of $10^{-5}$ an uncertainty of less than $3 \mathrm{mK}$ is required (whatever the pressure to be measured). It is therefore necessary to improve the uncertainty on the value of the gas temperature by temperature probes and its location. If the refractometer is used in relative mode, the only constraint is that the temperature between the reference state and the measurement state is the same to within $3 \mathrm{mK}$.

\subsection{Pressure stability}

In previous works [11], we were limited by the stability of the pressure due to the use of the commercial pressure regulator. The pressure resolution was then about $0.2 \mathrm{~Pa}$ at the atmospheric pressure. To reduce this pressure stability, we have installed and tested a DHI PG7607 pressure balance associated with a $20 \mathrm{~cm}^{2}$ piston-cylinder whose pressure expanded uncertainty is $0.20 \mathrm{~Pa}+8.0 \times$ $10^{-6} p$ This has led to reach the target pressure stability in Figure 4 and then allows us to determine the pressure by means of the refractometer with a stability of $\pm 2 \mathrm{mPa}$ over $20 \mathrm{~min}$.

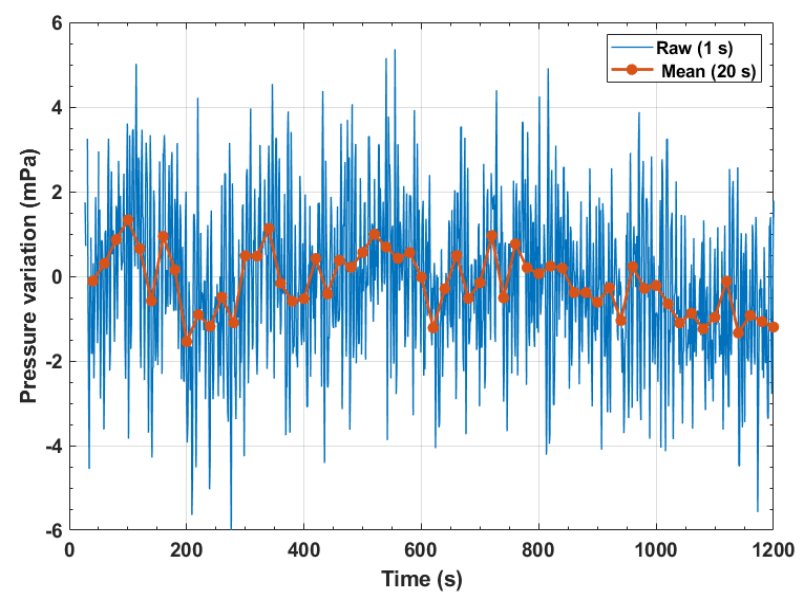

Figure 4: Pressure variation measured by the refractometer over $1200 \mathrm{~s}$.

We can note that this excellent result is only possible in the short term because, it is not possible to guarantee stability in pressure and temperature more than $20 \mathrm{~min}$.

\section{IMPROVED DESIGN OF THE REFRACTOMETER}

To resolve the main issues of the current refractometer, we have designed a new set-up still using a helium absolute refractometer at $532 \mathrm{~nm}$. This design has the advantage to be more compact (20 times smaller in volume) to avoid temperature gradient and temperature drift. Figure 5 presents a project design of the improved set-up: a $50 \mathrm{~mm}$ squared single Fabry-Perot resonator (two silica mirrors and a spacer made in Zerodur ${ }^{\circledR}$ ) and an enclosure in copper working under vacuum and gas.

We also will use encapsulated high quality $\mathrm{Pt} 25$ probe used at gallium point to have an uncertainty lower than $1 \mathrm{mK}$.

In the longer term, a FP cavity made of a more stable material than Zerodur ${ }^{\circledR}$ can be used. Several publications report a ceramic, NEXCERA ${ }^{\mathrm{TM}}$ (Krosaki Harima), which has an aging almost 5 times lower than Zerodur ${ }^{\circledR}$ [13]. 


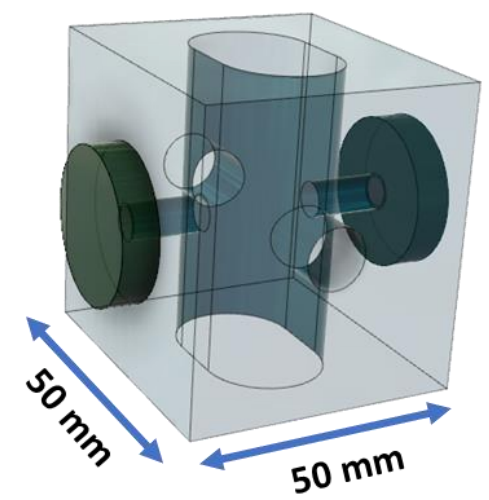

(a)

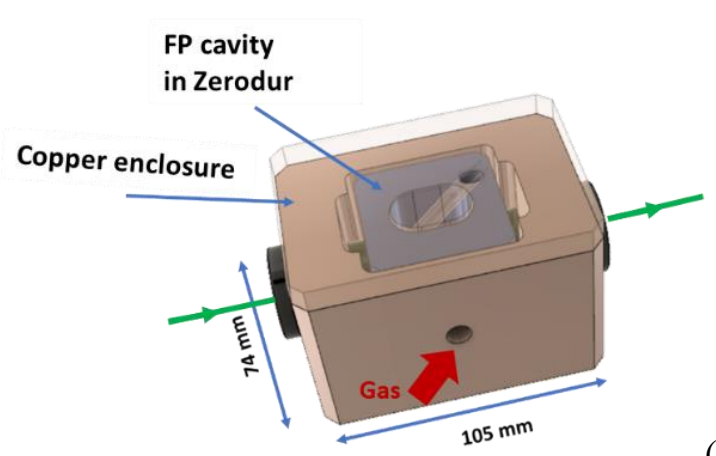

(b)

Figure 5: Project design of our new quantum pascal realization set-up. (a) $50 \mathrm{~mm}$ single Fabry-Perot (FP) resonator composed of two silica mirror and a spacer made in Zerodur ${ }^{\circledR}$. (b). Cross section of the copper enclosure including the FP resonator.

Simulations of the pressure-induced deformation have been performed on with new design of the Fabry-Pérot cavity using ANSYS Workbench Rxx academic version. We use the same methodology presented in [14]. The pressure-normalized deformation value is $\frac{\frac{\Delta L}{L}}{P}=-6.5810 \times 10^{-12} \mathrm{~Pa}^{-1}$. The simulated value for the current FP cavity presented in this paper is $\frac{\frac{\Delta L}{L}}{P}=-6.3899 \times$ $10^{-12} \mathrm{~Pa}^{-1}$.

\section{SUMMARY}

This paper describes a quantum realization of the pascal based a helium absolute refractometer at $532 \mathrm{~nm}$. The short-term stability in pressure is $\pm 2 \mathrm{mPa}$ with a resolution in pressure of this new optical pressure standard is better than $1 \mathrm{mPa}$ with temperature stability about $1 \mathrm{mK}$. Pressure can be determined using an absolute method or a relative method. Aging and pressure-induced deformation of the FP are the main contributions of the uncertainty in the relative mode. In the case of absolute mode, the thermodynamic temperature of the gas is also a limiting factor. For that, a new design of the refractometer is presented to have better temperature stability and accurate temperature measurements to reach uncertainties in pressure better than current conventional methods. Improvements (performance, uncertainties, etc.) are mainly linked to the objectives of the "QuantumPascal" EMPIR project.

\section{ACKNOWLEDGEMENTS}

This work is a part of QuantumPascal project (18SIB04) funded through the European Metrology Programme for Innovation and Research (EMPIR).

\section{REFERENCES}

[1] P. Gambette, R. M. Gavioso, D. M. Ripa, M. D. Plimmer, et L. Pitre, « Towards a Quantum Standard for Absolute Pressure Measurements in the Range $200 \mathrm{~Pa}$ to $20 \mathrm{kPa}$ Based on a Superconducting Microwave Cavity », in 2018 Conference on Precision Electromagnetic Measurements (CPEM 2018), Paris, 2018, , doi: 10.1109/CPEM.2018.8500912.

[2] D. Mari, M. Pisani, et M. Zucco, « Towards the realization of an optical pressure standard », Measurement, vol. 132, p. 402-407, 2019, doi: 10.1016/j.measurement.2018.09.069.

[3] K. O. Douglass et D. A. Olson, « Towards a standard for the dynamic measurement of pressure based on laser absorption spectroscopy », Metrologia, vol. 53, $\mathrm{n}^{\mathrm{o}} 3$, p. S96-S106, 2016, doi:10.1088/00261394/53/3/S96.

[4] K. Jousten et al., « Perspectives for a new realization of the pascal by optical methods », Metrologia, vol. 54, $\mathrm{n}^{\mathrm{o}} 6$, p. S146-S161, 2017, doi:10.1088/16817575/aa8a4d.

[5] P. F. Egan, J. A. Stone, J. K. Scherschligt, et A. H. Harvey, «Measured relationship between thermodynamic pressure and refractivity for six candidate gases in laser barometry », Journal of Vacuum Science \& Technology A, vol. 37, $\mathrm{n}^{\circ} 3$, p. 031603, 2019, doi:10.1116/1.5092185.

[6] P. F. Egan, J. A. Stone, J. E. Ricker, et J. H. Hendricks, «Comparison measurements of lowpressure between a laser refractometer and ultrasonic manometer », Review of Scientific Instruments, vol. 87, no 5, p. 053113, 2016, doi: 10.1063/1.4949504.

[7] O. Axner, I. Silander, C. Forssén, Y. Guo, et M. Zelan, « Gas modulation refractometry (GAMOR) On its ability to eliminate the influence of drifts », p. 15.

[8] I. Silander, T. Hausmaninger, C. Forssén, M. Zelan, et O. Axner, «Gas equilibration gas modulation refractometry for assessment of pressure with subppm precision », Journal of Vacuum Science \& Technology B, vol. 37, $n^{\circ} 4$, p. 042901, 2019, doi: 10.1116/1.5090860.

[9] Y. Takei, K. Arai, H. Yoshida, Y. Bitou, S. Telada, et T. Kobata, «Development of an optical pressure measurement system using an external cavity diode laser with a wide tunable frequency range ", Measurement, vol. 151, p. 107090, 2020, doi: 10.1016/j.measurement.2019.107090.

[10] Y. Yang et T. Rubin, «Simulation of pressure induced length change of an optical cavity used for optical pressure standard», Journal of Physics: 
Conference Series, vol. 1065, p. 162003, 2018, doi: 10.1088/1742-6596/1065/16/162003.

[11] Z. Silvestri, F. Boineau, P. Otal, et J.-P. Wallerand, «Helium-Based Refractometry for Pressure Measurements in the Range 1-100 kPa », in 2018 Conference on Precision Electromagnetic Measurements (CPEM 2018), 2018, p. 1-2, doi: 10.1109/CPEM.2018.8501259.

[12] T. Badr, S. Azouigui, J. P. Wallerand, et P. Juncar, «Absolute refractometry using helium», in Conference on Precision Electromagnetic Measurements (CPEM), 2010, p. 506-507, 2017.
[13] C. J. Kwong, M. G. Hansen, J. Sugawara, et S. Schiller, «Characterization of the long-term dimensional stability of a NEXCERA block using the optical resonator technique », Measurement Science and Technology, vol. 29, $\mathrm{n}^{\circ}$ 7, p. 075011, 2018, doi: 10.1088/1361-6501/aac3b0.

[14] J. Zakrisson et al., « SIimulation of pressure-induced cavity deformation - The 18SIB04 Quantumpascal project », présenté à IMEKO 24th TC3, 14th TC5, 6th TC16 and 5th TC22 International Conference, CavtatDubrovnik, Croatia, nov. 2020. 